das noch in Gluhen erhaltene Kupferoxyd feuchtes Sauerstoftyas geleitet, welches vorn durch eine Lösung von Silbersalpeter austrat. Es gelang das Austreiben des Jods nur unvollständig und langsam, daher ich die Methode als unpraktisch rerworfen habe. Erleichterte irgend eine Modification die Zersetzung des Kupferjodur's, so wäre das Jod zuerst auf das Silberblech zu übertragen, worauf man das jodfreie Kupleroxyd durch das hintere Ende des Rolires zu entfernen und das Silber vom aufgenommenen Jod durch Ueberleiten von Wasserstoff zu befreien hätte. Man erhielte so alles Jod in der vorgelegten Silberlösung. - Eine Bestimmung des Jods aus der Gewichtszunahme des Silbers ist nicht thunlich, da das Jodsilber schmilat und sich am Glase vertheilt.

Bestimmung des Chlors (Broms oder Jods) neben Cyan.

\title{
K. Kraut.
}

Bei Bestimmung des Chlors in organischen Substanzen nach $\mathrm{Ca}$ rius' Weise*) erschien es mir möglich, dass sich dem im Rohr ausgeschiedenen Chlorsilber Cyansilber beimengen könnte. Da iudess Cyansilber, wie ich gefunden habe, im zugeschmolzenen Rohre schon bei $100^{\circ}$ durch mehrständiges Erhitzen mit Salpetersäure von 1,2 spec. Gew. ind rasch bei höheren Temperaturen zersetzt wird, so ist die Befürchtung unbegründet. Aus dem angeführten Verhalten des Cyansilbers ergibt sich aber eine einfache Bestimmung des Chlors (Broms oder Jods) neben Cyan, welche demnach ganz so vorgenommen werden kann, wie Carius behufs Bestimmung dieser Elemente in organischen Verbindungen vorschreibt.

Laboratorium der polytechnischen Schule in Han nover, Juli 1863.

Ueber genaue Bestimmung des Eisens.
Von

Dr. F. Mohr.

Wir besitzen einen solchen Reichthum an maassanalytischen Methoden zur Bestimmung des Eisens, dass man bei der Auswahl in Ver-

*) Diese Zeitschrift 1. 240. 
legenheit ist. Diese Methoden gehen in zwei verschiedenen Richtungen, die eine besteht darin, dass man das Oxydul in Oxyd umsetzt, die andere darin, dass man das Oxyd in Oxydul überführt. Die erstere Reihe ist die ältere, und sie wird mit Chamäleon oder doppeltchromsaurem Kali ausgeführt. Diese beiden setzen die vollständige Reduction des Oxyds zu Oxydul voraus, and diess haben wir bis jetzt durch eine ansehnliche Zahl von Stoffen erreicht, nämlich 1) durch Zink, 2) durch schwetlige Säure, 3) durch Schwefelwasserstoff, 4) dureh Zinnchlorür, 5) durch Jodwasserstoff. Alle Reductionsmethoden leiden an dem gemeinschaftlichen Uebelstand, dass man kein sicheres Zeichen der vollendeten Reduction hat. Das einzige Salz, welches das Eisenoxyd und nicht das oxydul in saurer Lösung anzeigt, ist das Sulfocyankalium. Diess gibt aber mit jeder Eisenlösung im ersten Augenblick eine rothe Färbung. Löst man ganz reines schwefelsaures Eisenoxydul-Ammoniak in wenig Salzsäure und taucht einen mit Sulfocyankalium befeuchteten Glasstab ein, so erscheint im ersten Augenblick eine blutrothe Färbung, welche durch Umschütteln wieder verschwindet. Vermischt man das Gemenge des Eisensalzes und der Salzsäure mit Schwefelwasserstoffwasser und erhitzt es, so zeigt auch diese Flüssigkeit vorübergehend die rothe Färbung, ohne dasss man annehmen kann, dass Eisenoxyd darin gewesen sein könne. Auch eine mit Zinnchlorür reducirte Eisenchloridlösung, in welcher Jod und Stärke einen kleinen Ueberschuss von Zinnchlorür anzeigen, gibt die rothe Fürbung. Man kann mit allen Reductionsmitteln keine eisenhaltige Flüssigkeit darstellen, welche nicht dasselbe thut. Es ist demnach dieses Reagens ganz werthlos zu dem vorliegenden Zweck, und seine Anwendung lässt immer in Ungewissheit, ob eine vollständige Reduction stattgefunden habe. Man hat sich die Gewissheit desshalb immer durch andere Zeichen zu verschaffen gesucht, wie bei dem Zink durch die vollständige Farblosigkeit, eine sehr lange Zeit der Einwirkung, oder dadurch, dass sich die reducirenden Körper, $\mathrm{SO}_{2}$ und $J H$, noch aus der kochenden Elüssigkeit entwickelten, wo dann nach einiger Zeit wieder die Möglichkeit eintrat, dass von Neuem Oxydation stattgefunden habe.

Auf der anderen Seite haben wir bei der Ueberführung des Oxyduls in Oxyd in dem Ferrideyankalium ein ganz untrügliches Mittel zu erkennen, ob noch die kleinste Spur Eisenoxydul vorhanden sei, und es fällt auch hier die Einwirkung des atmosphärischen Sauerstoffes weg. Ich kam dadurch wieder auf die von mir angegebene Methode der Eisenbestimmung durch Zersetzung des Eisenchlorids mit Jodkalium und Messung des ausgeschiedenen Jods mit unterschwefligsaurem Kali 
zurück. Diese Methode hat nicht ganz die Billigung des Herausgebers dieser Zeitschrift (Bd. 1, 5. 27) gefunden, und es war nir diess eine Veranlassung, sie von Neuem einer scharten Prüfung zu unterwerten und sie so abzuändern, dass sie vielleicht jetzt als genügend genau und praktisch angesehen wird.

Die vollständige Oxydation des Oxyduls in salzsaurer Lösung geschieht durch Zusatz kleiner Mengen chlorsauren Kalis, welche in die kochende Lösung geworfen werden. Die Flüchtigkeit des Eisenchlorids macht man unschädlich, wenn man einen Trichter mit Glassplittern oder eine mit Glassplittern gefüllte sogenannte Chlorcalciumröhre aufsetzt, an deren unterer Spitze sich immer ein gelber Tropfen sammelt, der zurückfällt und abgespritzt werden kann. Zur Prüfung auf Eisenoxydul breitet man einen Tropfen Ferridcyankaliumlösung auf einem Porzellanteller aus und betupft diese Stelle mit der Spitze einer in die Eisenlösung ganz leicht eingetauchten Thermometerröhre. Sobald kein Blau erscheint, ist die Oxydation vollendet. Die Ferridcyankaliumlösung bereitet man am besten auf dem Teller selbst mit einigen Tropfen Wasser und etwas Pulver des Salzes. Die Menge der verbrauchten Eisenflüssigkeit ist verschwindend klein, 1 oder 2 Milligramm. Nachdem diese Probe bestanden ist, und kein Chlorgeruch mehr wahrgenommen wird, wirft man festes Jodkalium hinein, was jedenfalls genügend sein muss; um das Fisenchlorid ganz zu zersetzen. Das Glas verschliesst man sogleich mit einem Glasstopfen oder einem unten zugeschmolzenen Trichter; der mit kaltem Wasser getüllt ist. Es verdunstet dann keine Spur von Jod. Hat man Glasstöpselgläser mit mässig dickem Boden, so kann man die Auflösung der Eisenprobe, die Oxydation, die Zersetzung mit Jodkalium and die schliessliche Ausmessung des Jods in demselben Gefässe und ohne allen Verlust vornehmen. Man erhitzt diese Gläser auf einem Drahtnetz stehend und 3 bis 4 Zoll von der Flamme ohne alle Gefahr, und hält sie während der Jodausscheidung fest mit dem Stopfen geschlossen. Diese Zersetzung kann im Saudoder Wasserbad stattfinden und etwa ${ }^{1 / 2}$ Stunde dauern. Nach dieser Zeit ist die Zersetzung des Eisenoxyds so vollständig geschehen, dass, wenn man mit unterschwefligsaurem Natron die blaue Jodstärkefarbe weggenommen hat; eine fernere Erhitzung kein Bläuen mehr zur Folge hat. Man lässt vollständig erkalten, ehe man das Jod misst.

Gegen die frühere Art der Ausfïhrung unterscheidet sich die jetzige dadurch, dass immer eine mehr als genügende Menge Jodkalium angewendet wird, dass die vollständige Zersetzung vor dem Anfang der Messung geschehen ist, und dass diese erst nach der Abkühlung statt- 
findet. Es wird dadurch die Einwirkung der freien Salzsäure auf das unterschwefligsaure und das gebildete tetrathionsaure Natron ganz vermieden; es findet niemals eine Ausscheidung von Schwefel statt, und die Reaction der Stärke mit dem Jod ist bekanntlich bei niederen Temperaturen am schärfsten. Die Empfindlichkeit der Enderscheinung ist so gross, dass ein Tropfen zehntel unterschwefligsaures Natron schon zu gross ist, um die letzte Spur der Bläuung aufzuheben, so dass malı mit Theilen eincs Tropfens fortschreiten muss. Die Ablesung an der Burette mit Zehntel CC. genügt ebenfalls nicht der ungemeinen Schärfe des Farbenwechsels, jedoch kann man praktisch nicht weiter gehen. Es lässt sich wohl noch $1 / 2$ Zehntel CC. ablesen, aber niedere Brüche nicht mehr. Wenn bei Analysen hundertstel CC. vorkommen, so sind sie immer das Resultat einer Correction und nicht direct abgelesen. Büretten mit 100stel CC. sind so dünn, dass man sie 10 bis 12 mal füllen müsste, nm eine Analyse zu vollenden, wodurch aber wieder der Totalfehler der Bürette multiplicirt wird. Will man den Fehler vermindern, der aus den vernachlässigten Brüchen der Zehntel CC. entsteht, so muss man grössere Mengen Substanz in Arbeit nehmen. Der Fehler von $0,1 \mathrm{CC}$. bei Zehntelfüssigkeiten beträgt für Eisen 0,000 כ 6 Grm., und auf das Gewicht einer Probe von 0,56 Grm. ${ }^{1 / 1000}$ des ganzen Gewichts oder $1 / 10$ Procent. Es folgt daraus, dass die Ablesung mit Zehntel CC. 'sowohl für alle praktischen, als selbst für wissenschaftliche Fälle genügt, obgleich die Reaction noch weiter zu gehen erlaubte.

Das unterschwefligsaure Natron ist zum Ausmessen des Jods ausgezeichnet, jedoch nicht zur Grundlage, weil es Wasser enthält und auch von Spuren schwefelsaurer Salze und Mutterlauge nicht ganz frei ist. Die eigentliche Grundlage der ganzen Messung ist das geschmolzone doppelt chromsaure Kali. Nachdem sein Verhältniss zu metallischem Eisen sehr genau festgestellt ist (Titrirbuch, 2. Aufl., S. 216) und diess ganz genau mit dem Atomgewichte des Chroms 26,24, welches aus ganz anderen Versuchen abgeleitet war, stimmte, so haben wir hierin das einfachste und sicherste Mittel den Werth des unterschwefligsauren Natrons, welches in Lösung einer sehr unbedeutenden Veränderung unterworfen ist, festzustellen. Die Zehntelchromlösung enthält 4,919 Grm. des Salzes im Liter, und mit dieser Lösung muss die des unterschwefligsauren Natrons gleichwerthig im Volum sein. Man kann sie entweder stellen, oder nach der Titrestellung berechnen. Zur Titrestellung nehme man $20 \mathrm{CC}$. Chromlösung mit einer Pipette und bringe sie in eine Stöpselflasche, setze Salzsäure und Jodkalium zu, lasse ${ }_{1 / 2}$ Stunde warm stehen und bestimme dann das ausgeschiedene 
Jod. Es mitssen genan $20 \mathrm{CC}$. unterschwefligsaures Natron verbraucht werden. oder die etwa sonst verbrauchte Menge wird bei der Analyse immer gleich $20 \mathrm{CC}$. Zehntel-Lösung gesetzt und darnach bercchnet. Die Zahlen sind bei richtiger Flitssigkeit absolut gleich und bei unrichtigen proportional. Das Verschwinden der blauen Farbe der Jodstärlse wird von der bleibenden grünen Farbe des Chromchlorids nicht verdeckt und is entsteht dadurch nicht die geringste Unsicherheit. Die schon früher empfohlene Chlorzinkstärke hat sich als absolut haltbar bewährt. Verdünnte Flüssigkeiten stchen schon jahrelang in offenen Flaschen und im Sonnenlicht und sind noch nicht im geringsten verändert. Wenn die Flüssigkeit ganz klar filtrirt ist, so ist das Eintreten und Verschwinden der blauen Farbe selbst schöner und deutlicher, als bei Chamäleon.

Die Titrestellung des unterschwefligsauren Natrons durch Chromlösung ist in einfachster Form genau dieselbe, deren sich Bunsen schliesslich zur Bestimmung der Stärke seiner Jodlösung bedient hat, nur dass wir hier eine Messing statt einer Wägung, und eine blosse Mischung statt einer Destillation anwenden. Wäre die Chromlösung selbst zweifelhaft, so würde man doppelt chromsaures Kali abwiegen und in gleicher Art mit Jodkalium und Salzsäure zersetzen. 0,246 Grm. desselben müssen gleich $50 \mathrm{CC}$. unterschwefligsauren Natrons sein. Die Zehntel-Jodlösung, frisch bereitet, lässt allerdings nichts zu wünschen über; allein angebrochene Gläser zeigen sich immer schwächer im Gehalt, als frische, noch ganz gefüllte. Die Verdunstung von Jod beim Oeffnen der Gläser ist unvermeidlich.

Die Anwendung des doppelt chromsauren Kalis als Grundlage für die Messung des Eisens ist sicherer, als die Anwendung einer Eisenlösung selbst, da man bei letzterer die Unreinlichkeiten des metallischen Eisens nicht in Rechnung ziehen oder beseitigen kann, und wasserhaltige Salze immer weniger zuverlässig sind, als wasserleere, die Schmelzung vertragende.

Zur Bestimmung des Eisenoxyduls, was in der Probe selbst vor* handen ist, verdient die Chromlösung den Vorzug vor dem Chamäleon, sowohl wegen ihrer Titrebeständigkeit, der Verhältnissmässigkeit ihrer Mengen, als auch, weil sie mit verdünnter Salzsäure kein Chlor bildet. Salzsäure kann man bei Eisenerzen gar nicht vermeiden, und da sie nach Löw enthal and Lenssen (diese Zeitschrift Bd. 1. S. 329) einen Einfluss auf die Resultate hat, so ist dadurch dem Chamäleon eigentlich der Glanz genommen. Man kann mit Chamäleon anch nicht den Titre des unterschwefligsauren Natrons stellen und müsste zu diesem Zwecke 
immer doch die Chromlösung haben. Aus diesen Gründen entschlägt man sich des Chamäleons vollständig bei dieser Analyse.

Um eine der mannigfaltigen Anwendungen der Combination beider Methoden kennen zu lernen, müssen wir erst die Genauigkeit der verbesserten Methode aus Vergleichen er'sehen. Wir nehmen an, dass eine frisch bereitete Chromlösung und eine ihr gauz gleichgestellte unterschwefligsaure Natronlösung vorhanden sei.

20 CC. Chromlösung mit Jodkalium und Salzsäure 1/2 Stunde stehen gelassen, erforderten genau 20 CC. Zehntel $\mathrm{NaO}, \mathrm{S}_{2} \mathrm{O}_{2}$.

0,7 Grm. schwefelsaures Eisenoxydul-Ammoniak in Wasser gelösst, mit Salzsäure versetzt, erforderten, nach Schabus-Penny, 18 CC. Chromlösung. $18 \times 0,0056$ geben $0,1008 \mathrm{Grm}$. Eisen statt $0,1 \mathrm{Grm}$., welche in $0,7 \mathrm{Grm}$. des Salzes enthalten sind. Zuviel : 0,0008 Grm. Dieselbe Flüssigkeit mit Jodkalium digerirt, erforderte 18,1 CC. unterschwefligsaures Natron. Es konnten jetzt noch 2 Tropfen Chromlösung zugefügt weralen, der dritte Tropfen machte blau. 2 Tropfen sind 0,1 CC., so dass in beiden Fällen gleich viel Flüssigkeit verbrancht wurde.

Ein Stäck Eisendraht wog 0,163 Grm.; in Salzsäure gelöst, oxydirt und mit unterschwefligsanrem Natron gemessen, forderte $29,1 \mathrm{CC}$. ${ }_{1 / 10} \mathrm{NaO}_{\mathrm{S}_{2}} \mathrm{O}_{2}$. Diese machen $0,16296 \mathrm{Grm}$. Eisen aus. Differenz zu wenig: $0,00004 \cdot \mathrm{Grm}$.

Ein Stück Eisendraht $\operatorname{wog} 0,323$ Grm. Es erforderte 57,6 CC. $1 / 10 \mathrm{NaO}, \mathrm{S}_{2} \mathrm{O}_{2}$. Diess macht 0,32256 Grm.; Differenz zu wenig: $0,00044 \mathrm{Grm}$.

Zwei Stücke Eisendraht, gleich schwer gemacht, jedes 0,2082 Grm. wiegend, wurden einzeln gelöst, das eine mit chlorsaurem Kali oxydirt und nach dem Wegkochen des Chlors das andere hinzugefügt; erst wurde das Oxydul mit Chromlösung bestimmt und davon 37,1 CC. verbraucht. Das gibt 0,20766 Grm. Eisen als Oxydul; jetzt wurde Jodkalium zugefügt, $1_{2}$ Stunde digerirt und dann gemessen. Es wur(len 74,1 CC. $1 / 10$ NaO, $\mathrm{S}_{2} \mathrm{O}_{2}$ verbraucht. Davon abgezogen $37,1 \mathrm{CC}$. lassen $37 \mathrm{CC} .=0,2072 \mathrm{Grm}$. Eisen als Oxyd. Differenz im ersten Fall zu wenig: 0,00044 Grm., im zweiten Falle 0,001 Grm. Eisen.

Ein Krystall von Eisenoxyd-Ammoniakalaun wog 2,886 Grm., in Salzsäure gelöst und regelmässig gemessen, erforderte $59,5 \mathrm{CC}$. $1_{10} \mathrm{NaO}, \mathrm{S}_{2} \mathrm{O}_{2}$; diese mit 0,008 multiplicirt (nämlich $\mathrm{Fe}_{2} \mathrm{O}_{3},=80$ ), geben $0,4760 \mathrm{Grm}$. Eisenoxyd. Die Berechnung fordert $0,479 \mathrm{Grm}$; Differenz zu wenig : 0,003 Grm. Eisenoxyd. Diess war die stärkste $A b$ weichung von der Richtigkeit, allein auch bei einem krystallisirten Salze. Gleiche Mengen (10 CC.) Jodlösung wurden in 2 Gläser ge- 
than und der einen schwetelsaures Fisenoxydul-Ammoniak reichlich zugefügt. Beim Ausmessen mit $1 / 10 \mathrm{NaO} . \mathrm{S}_{2} \mathrm{O}_{2}$ erforderten beide ganz genau gleich viel; Eisenoxydul bindet also kein Jod in der Lösung.

Aus allen diesen Belegen geht herror, dass die Bestimmung des Eisens durch Jodkalium eine sehr grosse Schärfe und Genauigkeit des Resultats gibt. Aber ebenso leicht ist sie zu handhaben. Die Lösung der Eisenerze muss so wie so stattfinden; dieselbe Iösung ist genügend, um sowohl Oxydul, als Oxyd zu bestimmen, die Maassflüssigkeiten sind sehr haltbar, und die am mindesten haltbare bedarf in 3 Wochen keiner frischen Titrestellung, die übrigens sehr leicht auszuführen ist. $1 \mathrm{Grm}$. Jodkalium genügt für jede Eisenerzprobe von $0,56 \mathrm{Grm}$. Gewicht. Es sind mir auch schon mehrere Hüttenwerke bekannt, wo die Methode mit vollständiger Befriedigung angewendet wird. Diess spricht für die leichte Ausführbarkeit, während die Richtigkeit der Resultate oben nachgewiesen wurde.

Viele schlechte Braunsteine werden jetzt in die Eisenhochöfen gegeben. Dabei kommt es auf den Gehalt an Vangen, nicht auf seinen Sauerstoff an und zugleich auf den Eisengehalt. Beide Bestimmungen geschehen mit derselben Probe. 1 Grm. des Palvers wird im Platintiegel stark geglüht, um die Manganoxyde in $\mathrm{Mn}_{3} \mathrm{O}_{4}$ zu verwandeln; dann destillirt man die Probe mit Salzsäure in Jodkalium hinein und bestimmt das freie Jod. 1 CC. ${ }^{1 / 10} \quad \mathrm{NaO}, \mathrm{S}_{2} \mathrm{O}_{2}$ ist gleich $0,010671 \mathrm{Grm}$. Manganoxydul (MnO); der Rückstand im Kölbchen mit Jodkalium digerirt, gibt das Eisenoxyd. 1 CC. $=0,008 \mathrm{Grm}$. $\mathrm{Fe}_{2} \mathrm{O}_{3}$. Ferner kann man die einzelnen Oxydationsstufen des Mangans in seinen Erzen bestimmen, wenn man $1 \mathrm{Grm}$. ungeglüht, und $1 \mathrm{Grm}$. scharf geglüht. mit Salzsäure in Jodkalium destillirt (4 Minuten) und jedesmal das freie Jod mit unterschwefligsaurem Natron misst. Die ungeglühte Probe gibt den ganzen freien Sauerstoff (p). 1 CC. $=0,0008$ Grm. Sauerstoff. Die geglühte Probe gibt das Manganoxydul (m). 1 CC. $=$ 0,010671 Grm. Mno. Enthält nun der Braunstein x Manganhyperoxyd $\left(\mathrm{MnO}_{2}\right)$ und y Manganoxyd $\left(\mathrm{Mn}_{2} \mathrm{O}_{3}\right)$, so ist nach der in meinem Lehrbucke 2. Aufl., S. 510 abgeleiteten Formel

$$
\begin{aligned}
\mathrm{MnO}_{2} & =\frac{\mathrm{p}-0,101(\mathrm{~m}+\mathrm{p})}{0,0822} \\
\text { und } \mathrm{Mn}_{2} \mathrm{O}_{3} & =\mathrm{m}+\mathrm{p}-\mathrm{x} .
\end{aligned}
$$

Diese Analysen, die sonst zu den schwierigsten und verwickeltesten gehörten, sind durch Verbesserung der Methoden zum Spiele geworden.

Eigentlich praktisch ist die verbesserte Eisenbestimmungsmethode durch den Umstand, dass man keinen neuen Titre an jedem Tage zu 
nehmen hat, was meistens ebensoviel Zeit und Mühe kostet, als die Analyse selbst. Ich freue mich, dass ich in der Kritik ron Prof. Fresenius Veranlassung gefunden habe, der Methode die jetzige vollendete Form zu geben.

\section{Die Bestimmung der Phosphorsäure betreffend. Von}

\section{Dr. F. Mohr.}

Bei Analysen von Erden, Knochenmehl, Superphosphat, Guano erhält man in einem gewissen Stadium der Analyse einen Niederschlag von phosphorsaurem Eisenoxyd durch essigsaures Natron bei vorwaltender Phosphorsänre, über dessen Zusammensetzung man nicht einverstanden ist. Während die grösste Menge der Phosphorsüure mit Kalk verbunden in Essigsïure gelöst bleibt, scheidet sich der ganze Eisenoxydgehalt mit Phosphorsäure als ein grünlich weisser Niederschlag ab. Wenn dieser Niederschlag eine constante Zusammensetzung hat, so kann man aus dem Eisenoxydgehalt auf die Phosphorsäure schliessen, und da nun die so sehr leichte und sichere Bestimmung des Eisenoxyds (s. oben S. 243 ff.) zur Hand ist, so lag darin eine Veranlassung den Gegenstand noch einmal ins Auge zu fassen.

Ich hatte in der ersten Auflage des Titrirbuches (S. 225) diesen Niederschlag für ${ }^{5} / 4$ phosphorsaures Eisenoxyd mit der Zusammensetzung

$$
\begin{aligned}
& \text { 52,71 Phosphorsäure } \\
& \frac{47,29 \text { Eisenoxyd }}{100,000}
\end{aligned}
$$

erklärt. Nach der Analyse von Fresenius und Will*) hat ihn Pinkus für $1^{1 / 2}$ phosphorsaures Eisenoxyd angesprochen. Er sagt S. 104 seiner agriculturchemischen Versuche: $\gg$ da bei überschüssiger $\mathrm{PO}_{5}$ die Verbindung die constante Zusammènsetzung $\left(\mathrm{Fe}_{2} \mathrm{O}_{3}\right)_{2}\left(\mathrm{PO}_{5}\right)_{3}$ hat. $\ll$ Da Pinkus ein sehr zuverlässiger. Beobachter ist, so war darin eine Veranlassung gegeben, die Analyse zu wiederholen.

Es wurden demnach $0,2065 \mathrm{Grm}$. Eisendraht in Salzsäure gelöst, mit chlorsaurem Kali oxydirt, an 2 Grm. phosphorsaures Natron zugesetzt und mit einem Ueberschuss von essigsaurem Natron gefällt. Der Niederschlag gut gewaschen und geglüht wog 0,625 Grm. Obige 0,2065 Grm. Eisen gaben 0,295 Grm. Fisenoxyd, als Rest 0,330 Grm. Phos-

*) Annalen der Ohem. u. Pharm. 1844. Bd. 50, S: 379. 